\title{
OPEN Identifying environmental risk factors for inflammatory bowel diseases: a Mendelian randomization study
}

\author{
Robert Carreras-Torres ${ }^{1,2,3 凶}$, Gemma Ibáñez-Sanz ${ }^{2,3}$, Mireia Obón-Santacana ${ }^{1,2,3}$, \\ Eric J. Duell ${ }^{1,2}$ \& Victor Moreno $0^{1,2,3,4}$
}

Several studies have examined environmental factors and inflammatory bowel diseases (IBD) using traditional approaches; however, provided results are still conflicting. Our aim was to determine whether lifestyle and nutrient exposures, related to IBD in observational meta-analyses, influence IBD risk using a Mendelian randomization (MR) approach. A two-sample MR approach was applied on summary-level genome-wide association results. Genetic variants strongly associated with measures of tobacco smoking, obesity and fat distribution, physical activity, and blood levels of vitamins and fatty acids were evaluated on genetic data from international IBD consortia including a total of 25,042 IBD cases (12,194 cases of Crohn's disease (CD) and 12,366 cases of ulcerative colitis (UC)) and 34,915 controls. Our results indicated that, among lifestyle exposures, being a smoker was positively associated with CD (OR 1.13, $P=0.02$ ), but it was not associated with UC risk (OR 0.99, $P=0.88$ ). Bodymass index (BMI) and body fat percentage were positively associated with $C D(O R 1.11, P=0.02$, per standard deviation (SD) of $4.6 \mathrm{~kg} / \mathrm{m}^{2}$; and OR 1.50, $\mathrm{P}=3 \times 10^{-10}$, per SD of $6.6 \%$; respectively); while for UC, BMI was inversely associated (OR $0.85, P=5 \times 10^{-5}$; per SD) and body fat percentage showed a OR of 1.11 ( $P=0.11$; per $S D$ ). Additionally, among nutrient exposures, omega- 3 fatty acids levels were inversely associated with $C D\left(O R 0.67, P=2 \times 10^{-6}\right)$. Our MR results did not support a protective effect for being a smoker on UC risk; however, they are compatible with a risk effect for higher body fat proportion and a protective role for higher levels of omega-3 fatty acids on CD etiology.

Inflammatory bowel diseases (IBD) become chronic disorders as consequence of dysregulated immune response to intestinal dysbiosis in genetically susceptible individuals, with fluctuating periods between remission and relapses $^{1}$. There exist two main types of IBD, namely Crohn's disease (CD) and ulcerative colitis (UC). They differ, among other features, on the location of the affected mucosa; CD can affect discontinuously different regions of the gastrointestinal tract, typically ileum and colon, while UC starts in the rectum and may involve proximally the colon in a continuous fashion ${ }^{2,3}$.

IBD incidence and prevalence have stabilized in most economically developed areas, but continue to rise in areas in transition towards developed economies ${ }^{4}$. This links the IBD main risk factors to westernization of lifestyle. External environmental factors can contribute to gut dysbiosis, reducing microbiome diversity or introducing non-commensal microorganism, alter the integrity of the epithelial barrier, and trigger an altered immune response 5 . Environmental risk factors for gut dysbiosis and IBD are lifestyle parameters such as cigarette smoking, obesity, physical inactivity, and a western-type of diet (characterized by high intake of saturated fat, refined carbohydrates, red and processed meat, and low intake of fruits, vegetables, fiber, and fish $)^{6,7}$. A recent umbrella review of meta-analyses identified risk and protective factors with different levels of strength of epidemiologic evidence; however, subgroup analyses showed differential associations for factors such as smoking and folate levels ${ }^{7}$.

\footnotetext{
${ }^{1}$ Colorectal Cancer Group, ONCOBELL Program, Bellvitge Biomedical Research Institute (IDIBELL), Hospitalet de Llobregat, Avinguda de La Granvia de L'Hospitalet, 199, 08908 Barcelona, Spain. 2Unit of Biomarkers and Susceptibility, Oncology Data Analytics Program, Catalan Institute of Oncology (ICO). Hospitalet de Llobregat, Barcelona, Spain. ${ }^{3}$ Consortium for Biomedical Research in Epidemiology and Public Health (CIBERESP), Barcelona, Spain. ${ }^{4}$ Department of Clinical Sciences, Faculty of Medicine, University of Barcelona, Barcelona, Spain. ${ }^{\square}$ email: rcarrerastorres@gmail.com
} 


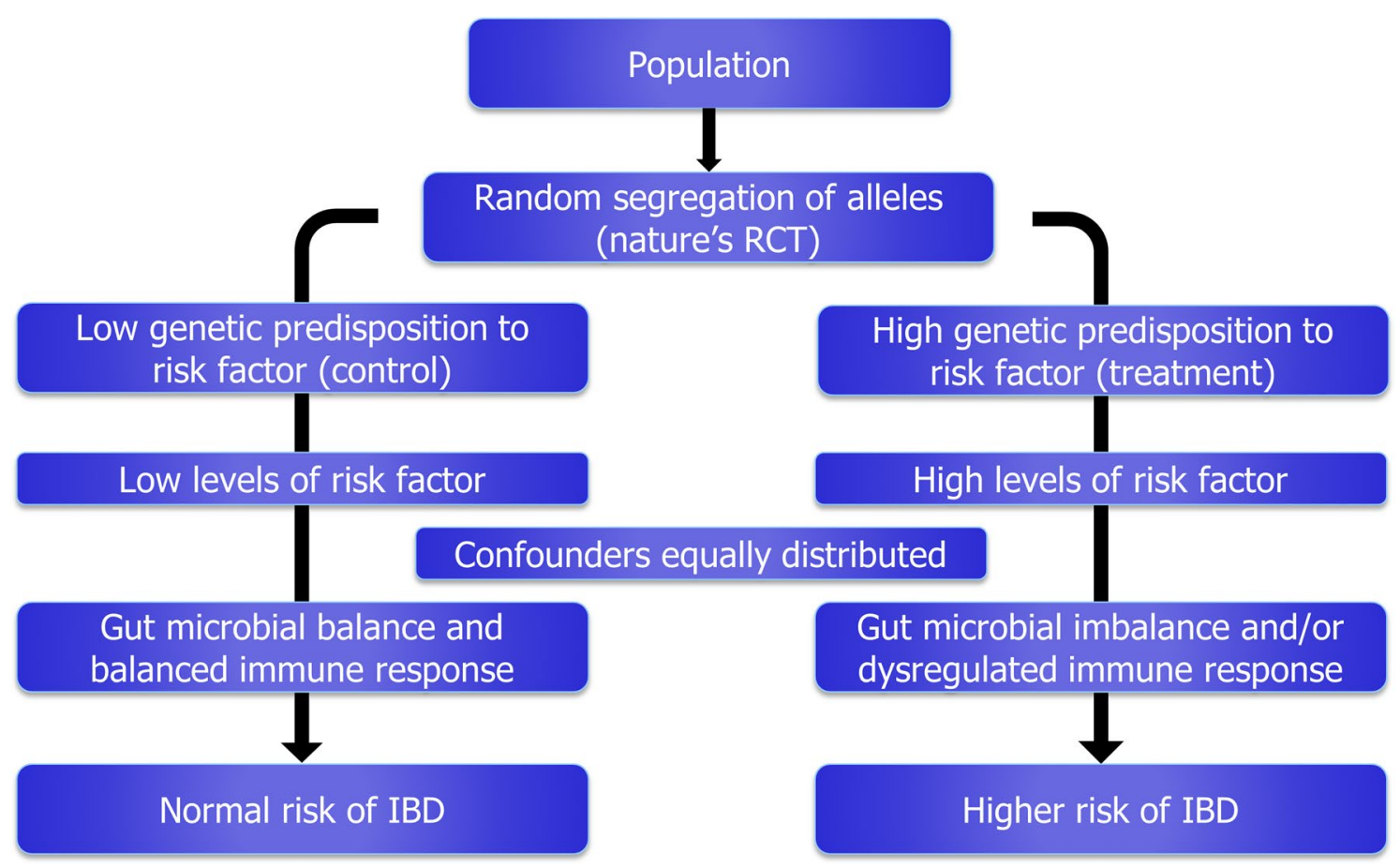

Figure 1. Mendelian randomization as an analogous approach to a classical randomized control trial (RCT).

Novel analytical methodologies aim to provide evidence of causality using genetic proxies for putative risk factors in instrumental variable analyses. Recently, one of these methods, called Mendelian randomization (MR), has become popular as large-scale genome-wide association studies (GWAS) allow the identification of genetic variants reliably associated with putative risk factors ${ }^{8}$. This methodology was proposed as analogous approach to a classical randomized controlled trial (RCT), the gold standard to establish causality. The random segregation of alleles at conception provides naturally two groups of population differentiated exclusively by the genetics of the putative risk factors (Fig. 1), mimicking the effect of the treatment and control arms in a RCT. Under the methodological assumptions, this approach avoids biasing effects from reverse causation (as disease onset does not affect germ-line genetics), and it is largely free from confounding (as the genetic basis of different risk factors are independent) $)^{9}$. The results from a MR approach allow a better estimation of the risk contribution of a putative risk factor, and support the development of preventive disease strategies.

In this study, for those environmental risk factors previously identified in large meta-analyses and with available GWAS data, we aimed to provide further evidence of potential causation using a two-sample MR approach. We reviewed and identified genome-wide variants associated with putative risk factors, and tested them on GWAS data from large-scale IBD international consortia comprising more than 50,000 individuals.

\section{Materials and methods}

This study was based on summary-level genome-wide association results under a two-sample MR approach ${ }^{10}$. Initially, genetic variants strongly associated with each exposure were identified as instruments in large-scale GWAS results (first sample). Subsequently, for the identified instruments, association data on IBD and subtypes were retrieved from IBD international consortia results (second sample). Genetics-to-exposure results were combined with genetics-to-IBD results to obtain exposure-to-IBD effect estimates through a likelihood-based MR approach. The consistency of initial results was evaluated by applying several complementary MR analyses.

Identification of genetic determinants for potential risk factors. Analyzed factors included measures of tobacco smoking, obesity and fat distribution, overall physical activity, and blood metabolite levels affected by a western-type of diet, such as vitamins and fatty acids.

Initially, genome-wide associated single nucleotide polymorphisms (SNPs) $\left(\mathrm{P}<5 \times 10^{-8}\right)$ were identified as genetic instruments in European-based studies. Recently published GWAS using or including the European individuals from the UK Biobank cohort are a rich source of genetic instruments. UK Biobank is a prospective cohort that recruited more than 500,000 men and women aged 40-96 years between 2006 and 2010, and collected anthropometric, health and lifestyle data ${ }^{11}$. The genetic etiology of tobacco smoking was recently described in a GWAS $^{12}$ comprising up to 1.2 million individuals from the Tobacco and Genetics (TAG) consortium, 23andMe database, and the UK Biobank cohort. From this study we identified genetic instruments for risk of ever being smoker (ever vs never smokers), and number of cigarettes smoked per day. Regarding obesity and fat distribution measures, genetic instruments for body-mass index $(\mathrm{BMI})^{13}$ and waist-to-hip ratio ${ }^{14}$ were retrieved from metaanalyses combining GWAS results from the Genetic Investigation of ANthropometric Traits (GIANT) consortium and the UK Biobank cohort comprising nearly 700,000 individuals. Genome-wide associated SNPs for body fat 


\begin{tabular}{|c|c|c|c|c|c|c|c|c|c|}
\hline \multirow[b]{2}{*}{ Potential risk factor } & \multirow[b]{2}{*}{ n SNP } & \multirow[b]{2}{*}{ Mean } & \multirow[b]{2}{*}{ SD } & \multirow[b]{2}{*}{ Units } & \multirow[b]{2}{*}{$\operatorname{Var}(\%)$} & \multirow[b]{2}{*}{ Pub ref } & \multicolumn{3}{|c|}{$\begin{array}{l}\text { Minimum } \\
\text { detectable OR }\end{array}$} \\
\hline & & & & & & & IBD & CD & UC \\
\hline \multicolumn{10}{|l|}{ Lifestyle } \\
\hline Smoking status & 331 & - & - & Ever/never & 2.3 & 12 & 1.17 & 1.22 & 1.21 \\
\hline Cigarettes per day & 44 & 2.35 & 0.94 & n cigarettes & 1.1 & 12 & 1.25 & 1.34 & 1.32 \\
\hline Body-mass index & 816 & 26.97 & 4.65 & $\mathrm{Kg} / \mathrm{m}^{2}$ & 6.0 & 13 & 1.10 & 1.13 & 1.13 \\
\hline Waist-to-hip ratio & 403 & 1 & 0.1 & - & 3.0 & 14 & 1.14 & 1.19 & 1.19 \\
\hline $\begin{array}{l}\text { Body fat percentage (fat/total } \\
\text { mass) }\end{array}$ & 378 & 31.8 & 6.6 & $\%$ & 3.5 & 15 & 1.13 & 1.18 & 1.17 \\
\hline Overall physical activity & 2 & 27.89 & 8.14 & Mili-gravities (acceleration) & 0.08 & 16 & 2.27 & 2.93 & 2.83 \\
\hline \multicolumn{10}{|l|}{ Nutrients } \\
\hline Vitamin D & 59 & 70.0 & 34.7 & $\mathrm{nmol} / \mathrm{l}$ & 3.4 & \begin{tabular}{|l|}
19 \\
\end{tabular} & 1.13 & 1.18 & 1.17 \\
\hline Vitamin B9 (folate) & 2 & 13.4 & 8.44 & $\mathrm{nmol} / \mathrm{l}$ & 1.0 & 20 & 1.26 & 1.36 & 1.34 \\
\hline Vitamin B12 & 10 & 342.68 & 150.11 & $\mathrm{pmol} / \mathrm{L}$ & 6.3 & 20 & 1.10 & 1.13 & 1.12 \\
\hline Omega-3 fatty acids & 4 & 0.41 & $0.08-1.80^{*}$ & $\mathrm{mmol} / \mathrm{l}$ & 2.4 & 21 & 1.16 & 1.22 & 1.21 \\
\hline Omega-6 fatty acids & 8 & 4 & $1.8-12.0^{*}$ & $\mathrm{mmol} / \mathrm{l}$ & 4.6 & 21 & 1.11 & 1.15 & 1.15 \\
\hline $\begin{array}{l}\text { Monounsaturated fatty } \\
\text { acids (mainly omega-9 and } \\
\text { omega-7) }\end{array}$ & 4 & 3.3 & $1.1-15.0^{*}$ & $\mathrm{mmol} / \mathrm{l}$ & 2.4 & 21 & 1.16 & 1.22 & 1.21 \\
\hline Total fatty acids & 8 & 11.7 & $4.6-42.0^{*}$ & $\mathrm{mmol} / \mathrm{l}$ & 3.7 & 21 & 1.13 & 1.17 & 1.17 \\
\hline
\end{tabular}

Table 1. Description of genetic instruments for observed environmental risk factors. $n S N P$ number of single nucleotide polymorphisms, $S D$ standard deviation, Var: explained phenotype variance, $P u b$ ref discovery GWAS, OR odds ratio; IBD Inflammatory bowel disease; $C D$ Crohn's disease; $U C$ ulcerative colitis. ${ }^{\star}$ Range (minimum-maximum).

percentage (total fat mass/ total mass) ${ }^{15}$ were identified from GWAS using nearly 360,000 UK Biobank individuals. Likewise, physical activity was recently genetically assessed in the UK Biobank cohort. Overall physical activity was measured using a wrist-worn accelerometer device in a subsample of 91,000 individuals ${ }^{16}$. Finally, we analyzed vitamins and fatty acids levels as metabolites affected by the type of dietary intake ${ }^{17,18}$. Genetic instruments were identified for circulating levels of vitamin $\mathrm{D}^{19}$, vitamin $\mathrm{B} 9$ and $\mathrm{B} 12^{20}$, polyunsaturated omega-3 and omega-6, monounsaturated (mainly omega-7 and omega-9) fatty acids, and total fatty acids ${ }^{21}$.

From the initial sets of SNPs, we selected uncorrelated SNPs based on linkage disequilibrium (LD) $R^{2}<0.01$. SNPs with ambiguous strand codification $(\mathrm{A} / \mathrm{T}$ or $\mathrm{C} / \mathrm{G})$ were replaced by SNPs in genetic linkage $\left(\mathrm{R}^{2}>0.8\right)$ using the proxysnps $\mathrm{R}$ package (European populations) (R Project). SNP-to-exposure association summary statistics were retrieved from the original GWAS studies, and can be observed in Supplementary Table S1 online. In the studies where summary statistics were not reported in standard deviations (SD), they were transformed based on SD reported in the original GWAS studies. Table 1 describes genetic instruments features on the number of SNPs included, the proportion of phenotype variance explained by the SNPs (or cumulative SNP liability in the case of binary outcomes), as well as the mean and SD of the respective exposure in the original GWAS studies.

GWAS data on inflammatory bowel diseases and subtypes. The largest GWAS on IBD and subtypes was based on meta-analyses results of European samples from the International IBD Genetics Consortium and the UK IBD Genetics Consortium ${ }^{22}$. This study comprised a total of 25,042 cases of IBD, including 12,194 cases of CD and 12,366 cases of UC, and 34,915 controls. SNP-to-IBD and subtypes association summary statistics for the selected SNPs were retrieved from this GWAS study, and can be observed in Supplementary Table S1 online.

Statistical analyses. Following the method proposed by Burgess ${ }^{23}$, a priori power calculations were performed for MR associations of nominal statistical significance $(\alpha<0.05)$. Power estimates depend on the phenotype variance explained by the genetic instrument, outcome sample size and case/control ratio. Minimum detectable odds ratios (OR) by the given genetic instruments and IBD samples can be observed in Table 1. In brief, most of the tested measures, 10 out of 13, showed enough power to detect moderate risk increases of $20 \%$ (minimum detectable OR $<1.20$ ) (Table 1). The analyses with less power were for overall physical activity, which genetic instrument explained $0.08 \%$ of phenotype variance, with a minimum detectable OR of 2.27 for IBD risk.

Estimated effects of each exposure on IBD, CD and UC outcomes reflected by each genetic variant (Wald estimates: $\beta_{\text {outcome }} / \beta_{\text {exposure }}$ ) were combined in a single estimate per outcome through a likelihood-based MR approach $^{10}$. This method assumes a linear relationship between the risk factor and outcome and a bivariate normal distribution for the genetic association estimates. Bonferroni multiple test correction was applied to consider evidence of exposure-to-IBD association (significance $=0.05 / 13$ : 0.0038). Heterogeneity between CD and UC risk effects was evaluated estimating the $\mathrm{P}$ value $\left(\mathrm{P}_{\text {disease het }}\right)$ for the $\mathrm{Q}$ statistic for heterogeneity, assuming a fixed-effect model of 1 degree of freedom. This MR approach is considered the most accurate method when the assumption of linear relationship between the exposure and the outcome (common assumption for the other 
methods as well) is held ${ }^{24}$. However, it is sensitive to invalid instruments due to pleiotropic SNP effects (i.e. SNP effects on the outcome independent from the proxied exposure: horizontal pleiotropy).

Several complementary MR approaches were applied to detect the presence of horizontal pleiotropy, outliers (SNPs with strong pleiotropy), and unbalanced horizontal pleiotropy of weak instruments. The initial sensitivity test is called MR pleiotropy residual sum and outlier (MR-PRESSO) test ${ }^{25}$. This method identifies heterogeneity between SNP effects $\left(\mathrm{P}_{\text {Global }}\right)$ as evidence of horizontal pleiotropy. Subsequently, it identifies outlier SNPs and provides evidence of biased effect on the initial risk estimates $\left(\mathrm{P}_{\text {Distortion }}\right)$. Another complementary MR method is called MR-Egger test ${ }^{26}$. This method identifies the presence of non-balanced horizontal pleiotropy in weak instruments (i.e. SNP effects biased towards one direction) in the intercept test of the regression analyses between SNP-to-exposure effects and SNP-to-outcome effects. The violation of this assumption (non-balanced pleiotropy) is critical for all other MR methods. Finally, to test the consistency of the initial risk estimates, two methods that rely on the density distribution of individual SNP exposure-to-outcome effects were applied, namely weighted median MR method ${ }^{27}$ and the modal-based MR estimate approach ${ }^{28}$. These methods estimate the weighed median and the mode, respectively, of this density distribution, and are less sensitive to the presence of invalid SNPs (SNPs with mild pleiotropic effect).

To visualize the SNP exposure-to-outcome effects and the presence of pleiotropic SNPs, scatter plots were used to depict the SNP-to-exposure and SNP-to-IBD effects, including the likelihood-based MR risk estimate. Statistical analyses were performed using $\mathrm{R}$ (The $\mathrm{R}$ project; $\mathrm{R}$ version 3.4.1), the TwoSampleMR (version 0.4.18) and MRPRESSO (version 1.0) R packages, and plots were generated using ggplot2 $\mathrm{R}$ package (version 3.1.0).

\section{Results}

Lifestyle exposures. Tobacco smoking was not robustly related to IBD risk. Initially, the genetic instrument for risk of ever being smoker was associated with a mild $13 \%$ risk increase for CD (95\% confidence interval (95\% CI) 1.02-1.25); while it was not for UC risk (OR 0.99, 95\% CI 0.90-1.10) $\left(\mathrm{P}_{\text {disease het }}=0.08\right)$ (Fig. 2). Smoking intensity (number of cigarettes smoked per day) showed OR of 1.17 (95\% CI 0.94-1.44, per SD of 1.1 extra cigarettes) and 0.90 (95\% CI 0.73-1.10, per SD) for CD and UC, respectively (Fig. 2). In the sensitivity analyses, MR-PRESSO test detected horizontal pleiotropy $\left(\mathrm{P}_{\mathrm{Global}}<8.7 \times 10^{-3}\right)$ and potential outlier SNPs; however, the presence of outlier SNPs did not bias the initial risk estimates $\left(\mathrm{P}_{\text {Distortion }}>0.19\right)$. MR Egger test detected evidence of directional pleiotropy for smoking intensity and CD risk $\left(\mathrm{P}_{\text {Egger intercept }}=4 \times 10^{-3}\right)$, and provided an OR of 0.73 (95\% CI 0.41-1.28, per SD). The other MR sensitivity approaches provided association estimates around the null for smoking status and smoking intensity on CD and UC risk (Supplementary Table S2 online). The scatter plots for the MR smoking status-to-CD association and smoking status-to-UC association are included in Fig. 3, while the other scatter plots can be observed in Supplementary Fig. S1 online.

We initially estimated that each SD increase in body-mass index (BMI; $\left.4.6 \mathrm{~kg} / \mathrm{m}^{2}\right)$ and waist-to-hip ratio $(0.1)$ were positively associated with CD risk (OR 1.11, 95\% CI 1.02-1.20; and OR 1.22, 95\% CI 1.09-1.37; respectively), but were inversely associated with UC risk (OR 0.85, 95\% CI 0.78-0.92; and OR 0.90, 95\% CI 0.81-1.01; respectively) $\left(\mathrm{P}_{\text {disease het }}<2.1 \times 10^{-4}\right)$ (Fig. 2). On the contrary, each SD increase in body fat percentage $(6.6 \%)$ was positively associated with CD risk (OR 1.50, 95\% CI 1.32-1.71) and UC risk (OR 1.11, 95\% CI 0.98-1.25) $\left(\mathrm{P}_{\text {disease het }}=7.3 \times 10^{-4}\right)$ (Fig. 2). Horizontal pleiotropy and potential outliers were observed in all the cases, however without biasing the observed risk estimates $\left(\mathrm{P}_{\text {Global }}<1 \times 10^{-4}, \mathrm{P}_{\text {Distortion }}>0.08\right)$ (Supplementary Table S2 online). Directional pleiotropy was not observed $\left(\mathrm{P}_{\text {Egger intercept }}>0.02\right)$, and the other MR sensitivity approaches provided similar relative risk estimates (Supplementary Table S2 online). The scatter plots depicting the MR BMI-to-UC association and body fat percentage-to-CD association are included in Fig. 4 . The other scatter plots can be observed in Supplementary Fig. S1 online.

Overall physical activity showed an inverse association with IBD risk (OR 0.51, 95\% CI 0.27-0.98, per SD of 8.1 miligravities of acceleration), without heterogeneity between $\mathrm{CD}$ and $\mathrm{UC}\left(\mathrm{P}_{\text {disease het }}=0.51\right)$ (Fig. 2$)$. However, SNP heterogeneity was observed for $\mathrm{CD}\left(\mathrm{P}=1.5 \times 10^{-3}\right)$, which was significant for a 2-SNP genetic instrument (Supplementary Table S2 online).

Nutrient exposures. One SD increase in circulating vitamin D levels $(34.7 \mathrm{nmol} / \mathrm{l})$ showed null association with IBD risk (OR 0.96, 95\% CI 0.87-1.06), without heterogeneity between CD and UC $\left(\mathrm{P}_{\text {disease het }}=0.85\right)($ Fig. 5). Horizontal pleiotropy and potential outliers were observed, however without biasing the observed risk estimates $\left(\mathrm{P}_{\text {Global }}<1 \times 10^{-4}, \mathrm{P}_{\text {Distortion }}>0.77\right)$ (Supplementary Table S2 online). MR vitamin D-to-IBD scatter plot can be observed in Fig. 6a. Regarding B vitamins, one SD increase in circulating vitamin B9 levels (8.44 nmol/l) showed an OR of 0.83 (95\% CI 0.67-1.03) for IBD ( $\left.\mathrm{P}_{\text {disease het }}=0.59\right)$ (Fig. 5); however, SNP heterogeneity was observed for the 2-SNP instrument $\left(\mathrm{P}=2.5 \times 10^{-3}\right.$; Supplementary Table S2 online). One SD increase in vitamin B12 levels $(150.11 \mathrm{pmol} / \mathrm{L})$ was initially associated with CD (OR 1.10, 95\% CI 1.00-1.21) and UC (OR 1.06, 95\% CI 0.971.15) $\left(\mathrm{P}_{\text {disease het }}=0.52\right)$ (Fig. 5). However, MR-PRESSO test detected horizontal pleiotropy $\left(\mathrm{P}_{\text {Global }}<1 \times 10^{-4}\right)$ and an outlier SNPs slightly biasing the initial risk estimate for $C D\left(\mathrm{P}_{\text {Distortion }}=0.02\right)$, while the other sensitivity $M R$ analyses provided null estimates for the vitamin B12-to-IBD and subtypes associations (OR from 0.92 to 1.09) (Supplementary Table S2 online). Scatter plots can be observed in Supplementary Fig. S1 online.

Finally, we observed that omega-3 fatty acid levels were inversely associated with IBD (OR 0.78, 95\% CI $0.68-0.88$, per SD of $\sim 0.20 \mathrm{mmol} / \mathrm{l})$. Some heterogeneity was observed between CD and UC $\left(\mathrm{P}_{\text {disease het }}=0.02\right)$, with ORs of 0.67 (95\% CI 0.56-0.79) and 0.88 (95\% CI 0.75-1.03), respectively (Fig. 5). The other analyzed fatty acid measures did not show association with IBD (OR 0.96, 95\% CI 0.88-1.05, for omega- 6 fatty acids; OR $0.94,95 \%$ CI $0.82-1.08$, for monounsaturated fatty acids (mainly omega-7 and omega-9); and OR $0.94,95 \%$ CI 0.85-1.04, for total fatty acids) (Fig. 5). Neither outlier SNPs, nor horizontal or directional pleiotropic effects were observed in these analyses. Additionally, the other MR methods provide similar relative risk estimates 


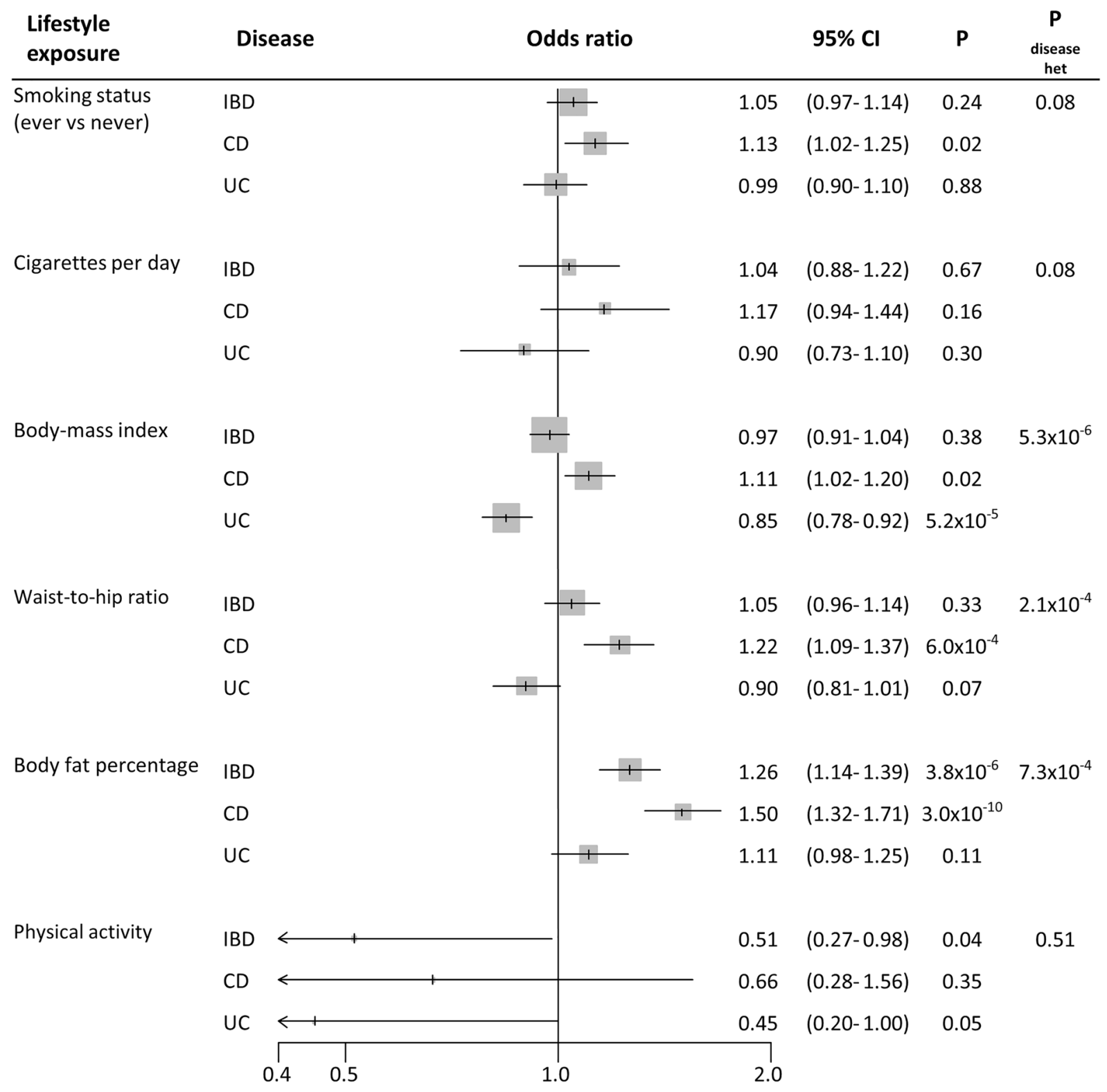

Figure 2. Forest plot of IBD risk for increment in lifestyle exposures. OR per standard deviation increase were estimated using likelihood-based MR approach. 95\% CI 95\% confidence interval; $P$ P value. $P_{\text {disease het }} \mathrm{P}$ value of heterogeneity between Crohn's disease (CD) and ulcerative colitis (UC).

(Supplementary Table S2 online). The scatter plot for the MR omega-3 fatty acids-to-CD relationship is included in Fig. 6b, while the other scatter plots can be observed in Supplementary Fig. S1 online.

\section{Discussion}

In this Mendelian randomization study, we found no evidence for a robust role of smoking on IBD and subtypes etiology. On the contrary, our results support an increased risk for higher body fat proportion and a protective role for higher levels of omega-3 fatty acids on CD etiology, as observed in large meta-analyses.

The most consistently reported potential risk factor for CD is tobacco smoking, showing risk increases higher than $50 \%$. On the contrary, the role of smoking on UC has been observed as protective with similar strength ${ }^{7}$. Our MR study is powered enough to validate the described associations, but we only observed a potential relative risk increase of $13 \%$ for ever being smoker on CD risk; with inconclusive results in the MR sensitivity analyses. Therefore, the observed associations in traditional meta-analyses could be confounded by other factors associated with both smoking and IBD subtypes. Supporting this evidence, there are some studies describing a null association with other types of nicotine consumption (moist snuff ${ }^{29}$ or in non-European populations $\mathrm{s}^{30}$.

There is considerable evidence that links obesity and IBD risk; a considerable proportion of IBD patients are obese, the incidence of both obesity and IBD is increasing, and obesity strongly modifies gut microbiota ${ }^{31,32}$. Despite this, findings from cross-sectional and cohort studies are conflicting; cross-sectional studies showed 


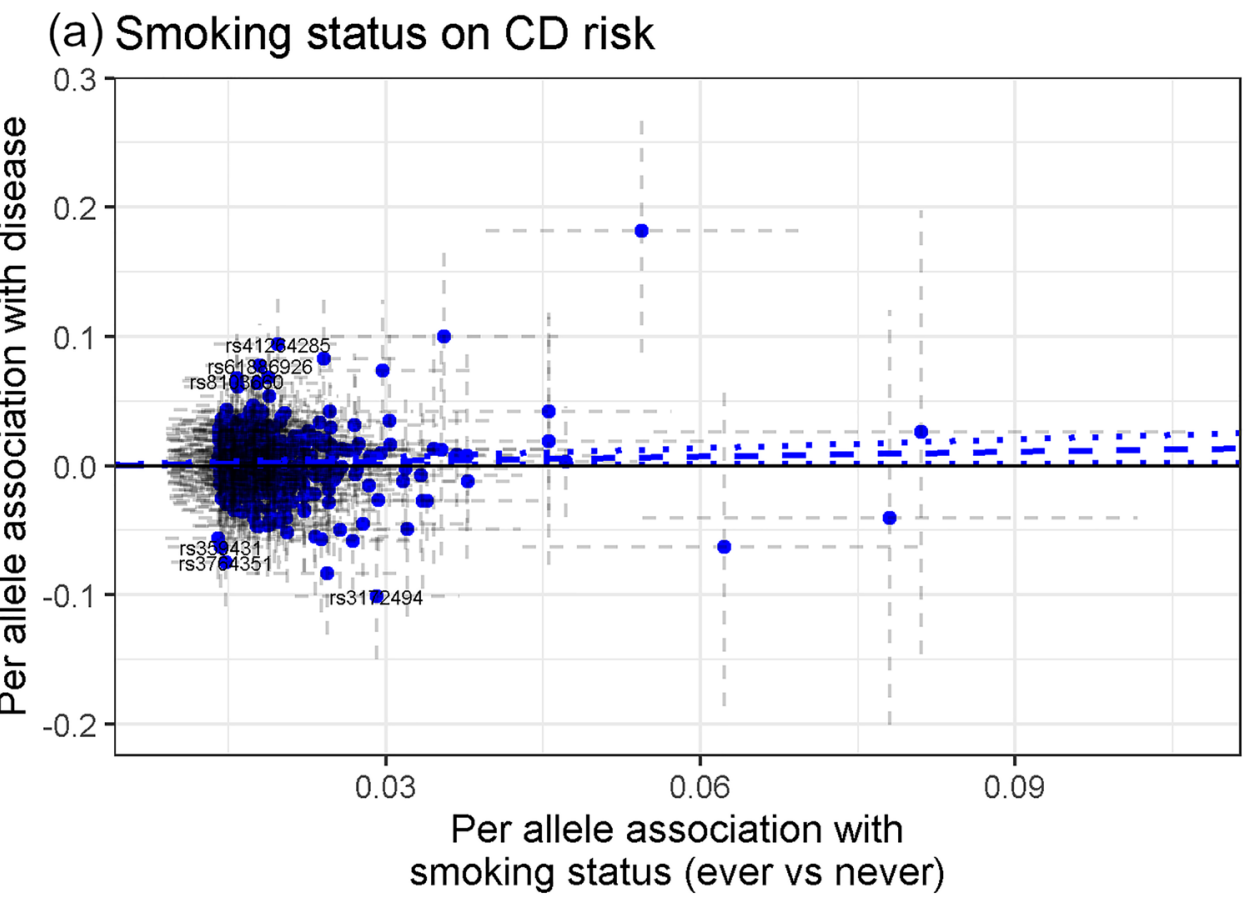

(b) Smoking status on UC risk

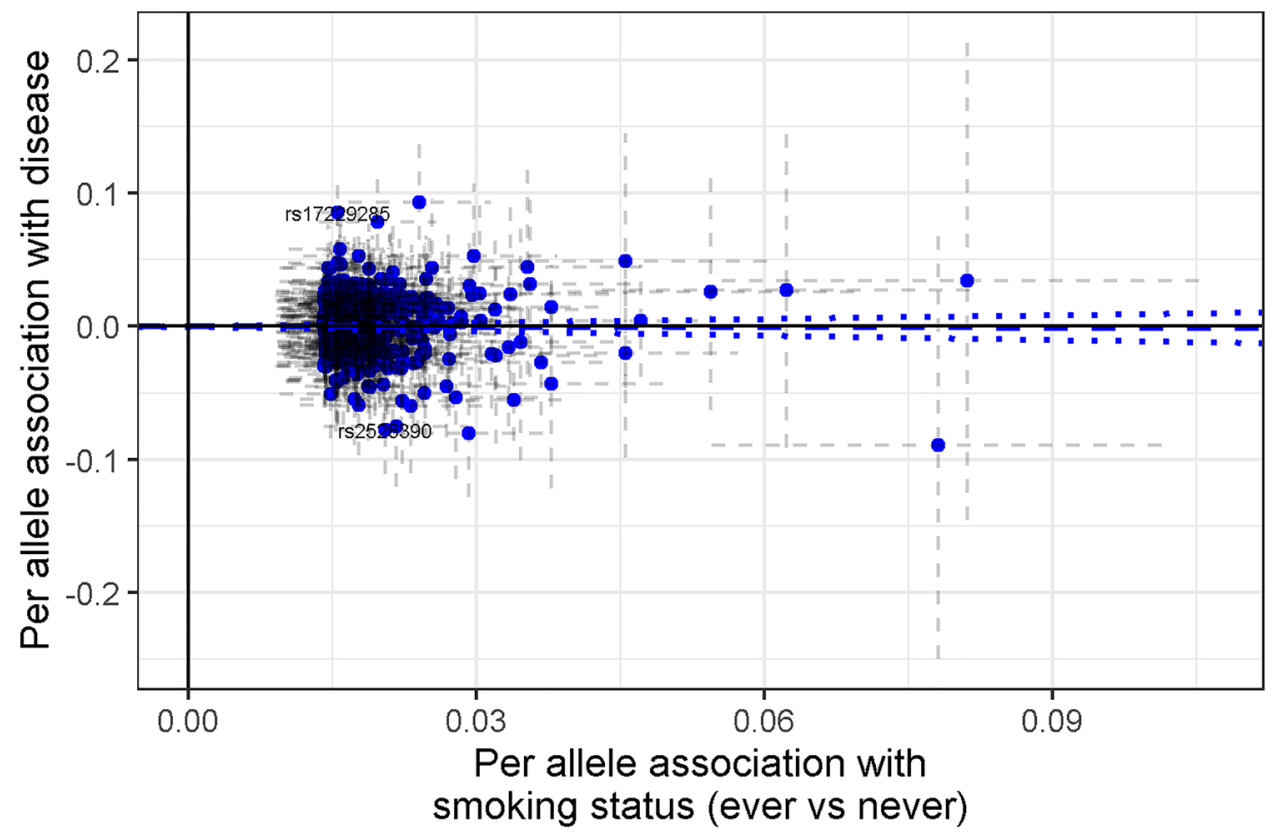

Figure 3. Scatter plots depicting summary statistics for genetic association and likelihood-based MR results of smoking status on CD (a), and smoking status on UC (b).

an inverse association between BMI and IBD and subtypes ${ }^{7}$, while cohort and population-based studies have described a positive association between $\mathrm{BMI}$ and $\mathrm{CD}$, but a negative trend between BMI and $\mathrm{UC}^{31,33}$. The present MR study is supporting the observations from cohort-based studies, and reveals a potential effect of reverse causation in the results from cross-sectional studies. Additionally, this opposing effect of BMI on CD and UC is similar to the reported pattern between being smoker and IBD subtypes in observational studies. Recently, a MR study analyzing obesity measures and smoking habits in more than 300,000 individuals identified higher levels of obesity as a factor increasing the risk for ever being a smoker and smoking at higher intensity ${ }^{34}$. Therefore, we hypothesize that the traditionally observed relation between smoking and IBD subtypes could be confounded by obesity, specially BMI. Finally, our MR results also confirmed that fat proportion would be more predictive for developing IBD, specially CD, than general obesity measures ${ }^{31}$. 
(a) Body-mass index on UC risk

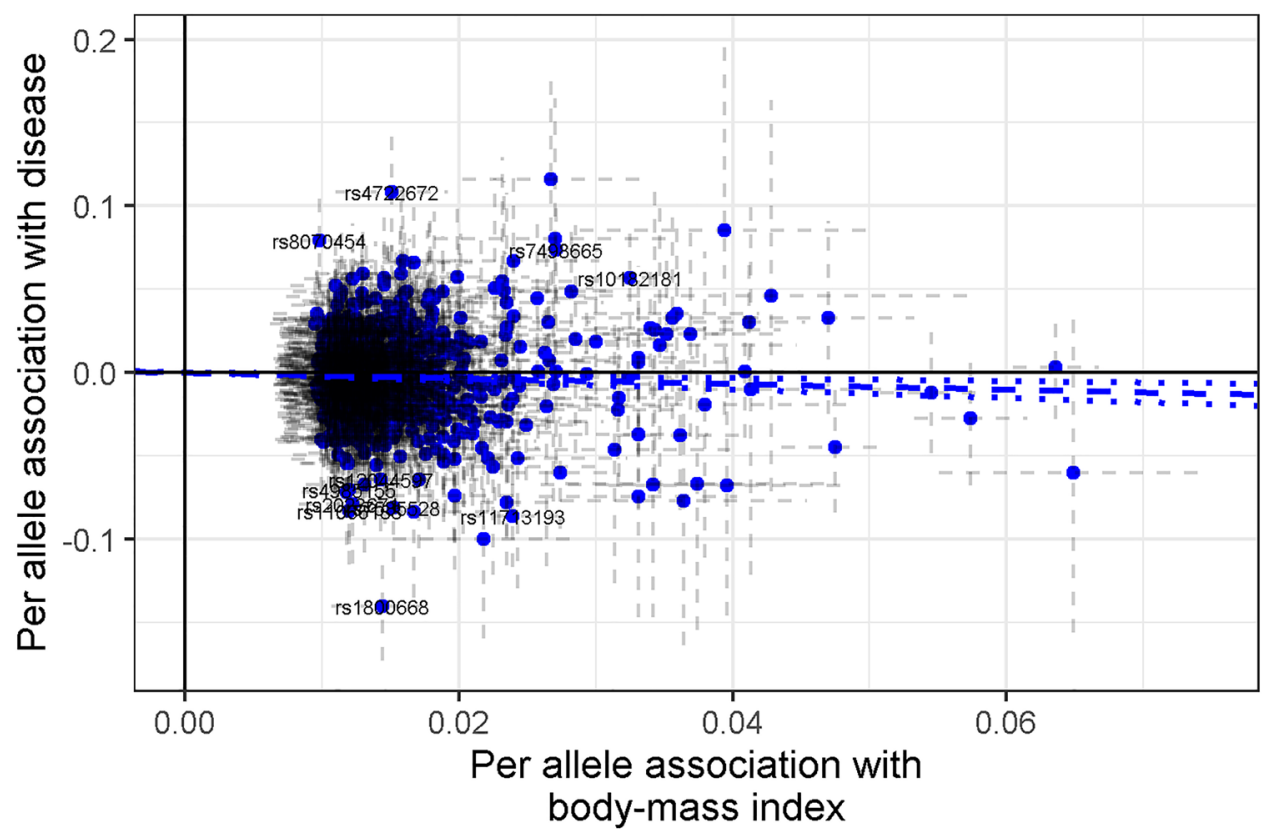

(b) Body fat percentage on CD risk

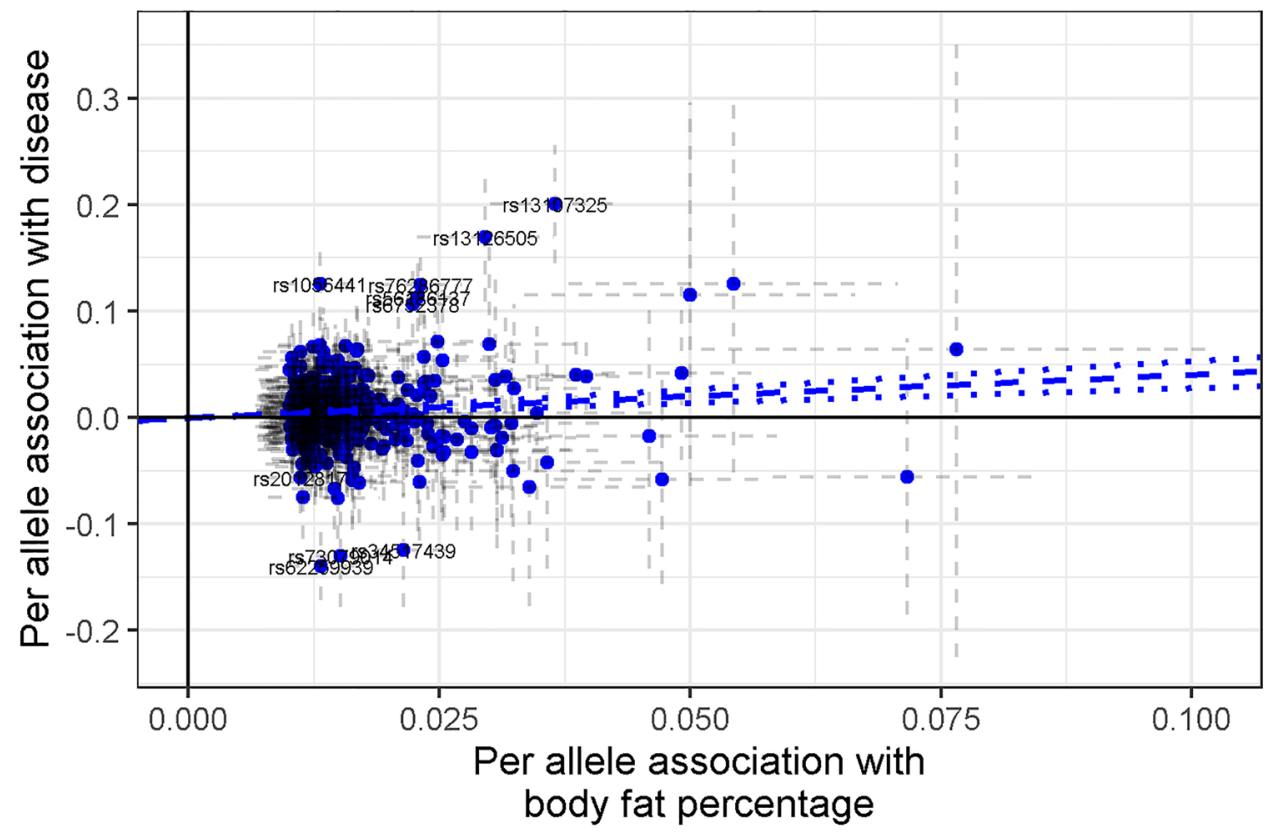

Figure 4. Scatter plots depicting summary statistics for genetic association and likelihood-based MR results of body-mass index on UC (a), and body fat percentage on CD (b).

Physical activity showed a robust inverse association with IBD and subtypes in large meta-analyses ${ }^{7}$. One limitation of the present MR study is the low statistical power for the overall physical activity genetic instrument (2 SNPs explaining the $0.08 \%$ of phenotype variance). Despite this, our MR estimate would be compatible with a protective effect of physical activity on IDB and subtypes risk.

Westernization of diet, characterized by high levels of saturated fat, red and processed meat instead of fish, and vitamin deficiency, is thought to be partially behind the rise in IBD incidence ${ }^{35}$. Both higher levels of D and B9 vitamins have been observed to reduce the IBD risk by $30 \%$ or more in large meta-analyses ${ }^{7,36}$. In a previous MR study on vitamin D, an association with IBD risk was not found; however this study included approximately 2,000 IBD cases $^{37}$. Our well-powered MR study, including 25,042 IBD cases, would not be consistent with a protective effect for higher levels of vitamin D and folate on IBD and subtypes risk. Regarding fatty acids, omega-3 fatty acids have been associated with a reduced risk, while omega- 6 fatty acids are considered a risk factor; however, 


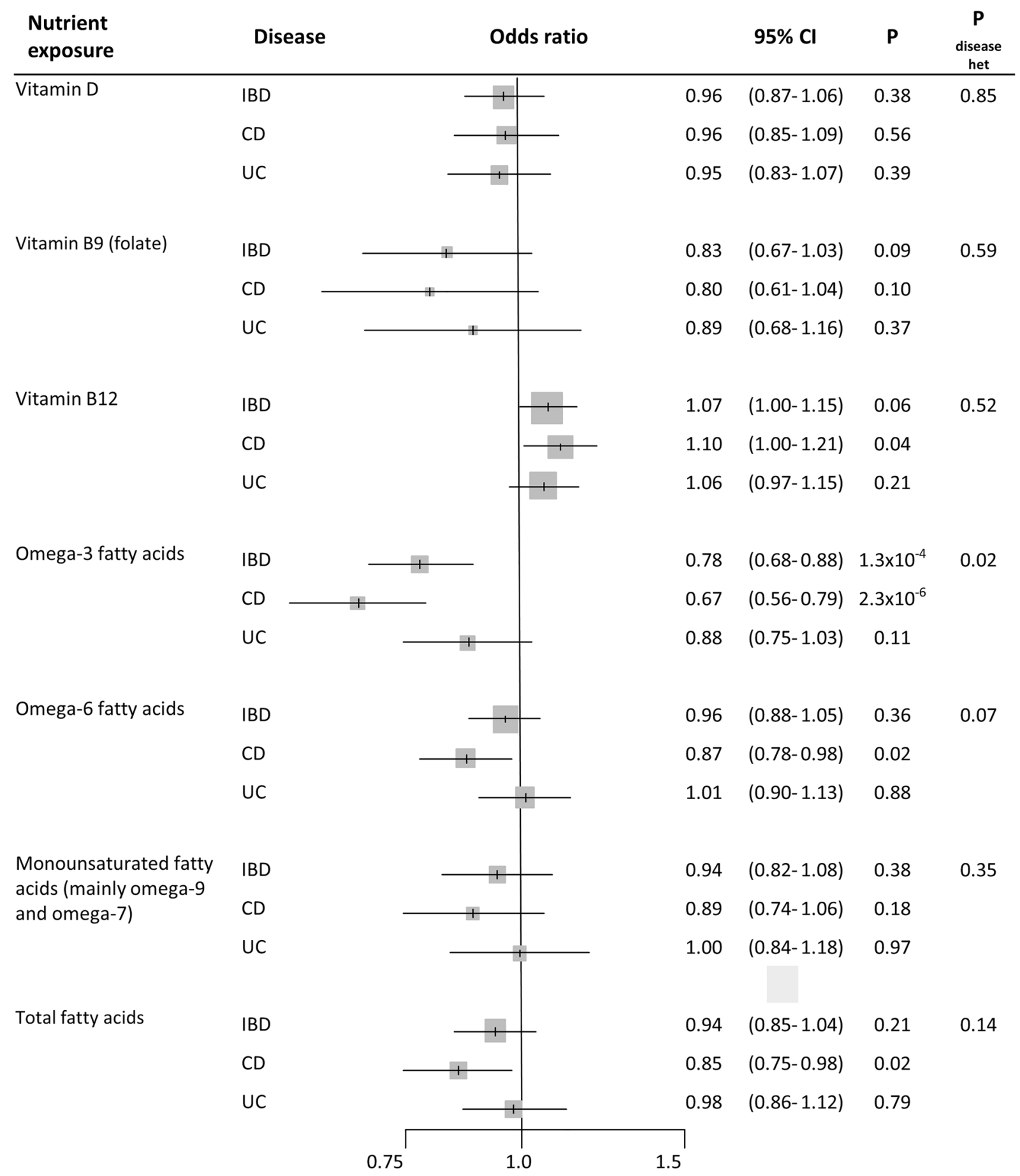

Figure 5. Forest plot of IBD risk for increment in nutrient exposures. OR per standard deviation increase were estimated using likelihood-based MR approach. 95\% CI: 95\% Confidence Interval; P: P value. $\mathrm{P}_{\text {disease het }}: \mathrm{P}$ value of heterogeneity between Crohn's disease (CD) and ulcerative colitis (UC).

there is no clear evidence of omega-3 therapeutic efficacy ${ }^{38-41}$. Our MR study provided evidence for a reduction of $\mathrm{CD}$ risk with higher levels of omega- 3 fatty acids. The contribution of omega- 3 reducing the CD risk would be mediated by their contribution to provide pro-resolving molecules in inflammatory processes ${ }^{41}$.

Despite limitations already mention, the main limitation of this study is the lack of analyses for putative risk factors related with living spaces, pre and post-natal, and birth conditions, surgeries, drugs, and microbiota risk factors, also observed in large meta-analyses ${ }^{7}$. For most of those factors, genetic instruments were not available because of the lack of genetic predisposition for those exposures. Recently, it has been assessed under a MR approach the contribution of microbial related traits (fecal propionate levels and butyrate gut production) on several metabolic parameters ${ }^{42}$. These genetic instruments did not reach our selection parameters, and we considered them unreliable. Studies with larger sample sizes are needed to identify microbial genetic instruments. 


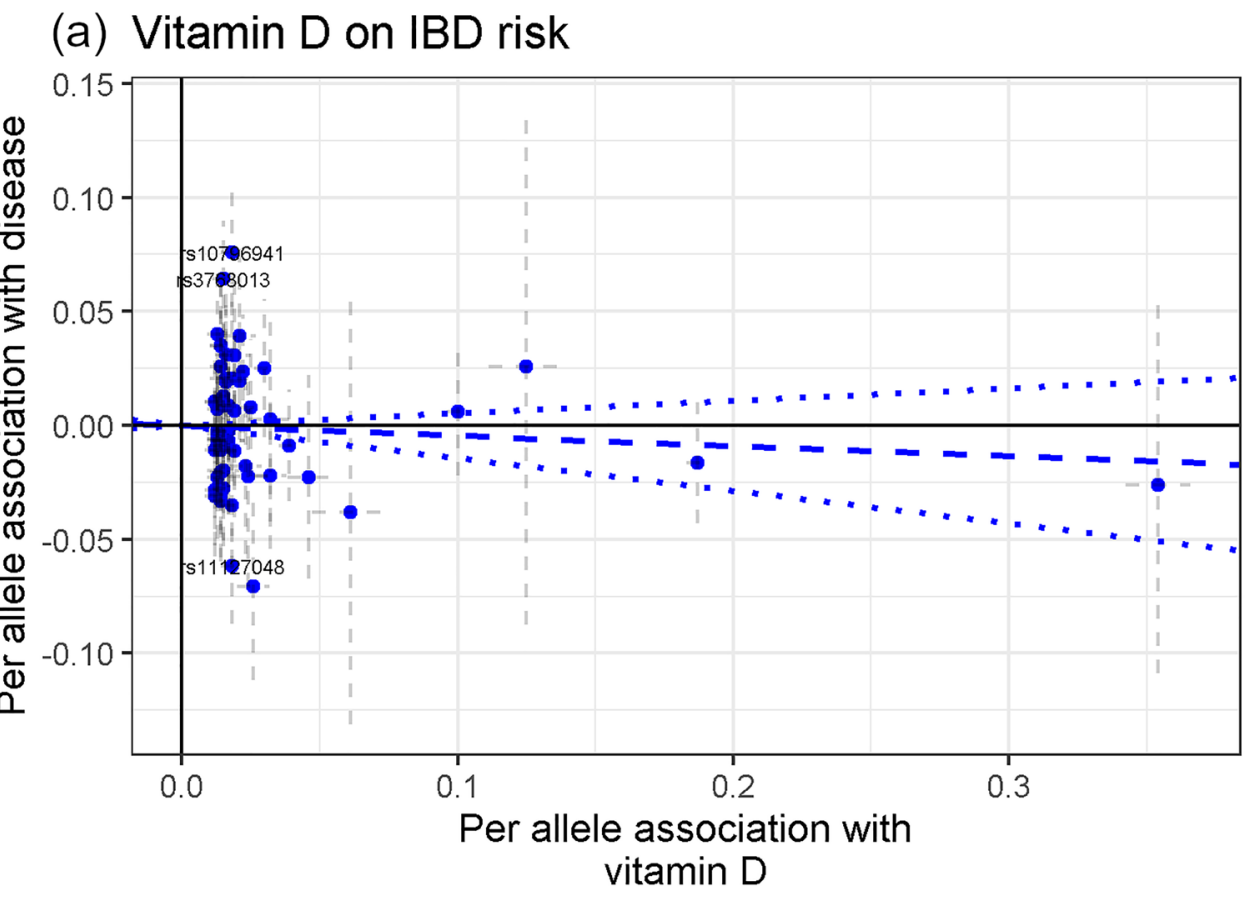

(b) Omega-3 fatty acids on CD risk

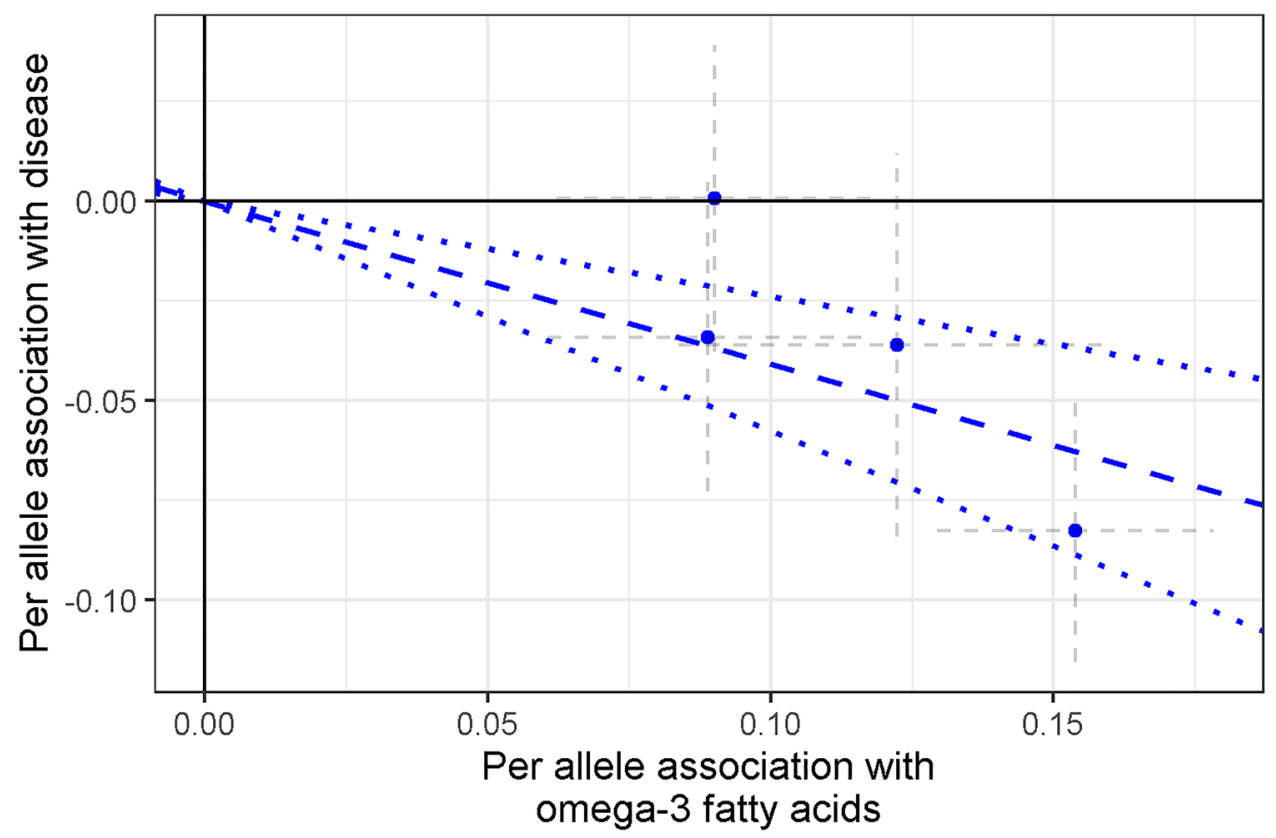

Figure 6. Scatter plots depicting summary statistics for genetic association and likelihood-based MR results of vitamin D on IBD (a), and omega-3 fatty acids on CD (b).

Finally, another limitation of this MR study is the potential violation of MR assumptions regarding pleiotropic effects on the analyzed genetic instruments; however, MR sensitivity analyses and graphical scatter plots were used to avoid biases of this nature.

In conclusion, our MR results did not support a robust role of smoking on IBD and subtypes etiology; however, they were compatible with a risk effect for higher body fat proportion and a protective effect for higher levels of omega-3 fatty acids on CD etiology, observed in meta-analyses. These results provided a better estimation of the contribution of risk factors to IBD etiology, and supported the development of preventive disease strategies related to physical activity and diet. 


\section{Data availability}

The dataset needed to reproduce the results is found in the Supplementary Table S1 online. URL for GWAS on IBD and subtypes: ftp://ftp.sanger.ac.uk/pub/project/humgen/summary_statistics/human/2016-11-07/.

Received: 28 January 2020; Accepted: 23 October 2020

Published online: 06 November 2020

\section{References}

1. Abraham, C. \& Cho, J. H. Inflammatory bowel disease. N. Engl. J. Med. 361, 2066-2078 (2009).

2. Gomollón, F. et al. 3rd European evidence-based consensus on the diagnosis and management of Crohn's disease 2016: Part 1: Diagnosis and medical management. J. Crohn's Colitis 11, 3-25 (2017).

3. Magro, F. et al. Third European Evidence-based Consensus on Diagnosis and Management of Ulcerative Colitis. Part 1: definitions, diagnosis, extra-intestinal manifestations, pregnancy, cancer surveillance, surgery, and Ileo-anal pouch disorders. J. Crohn's Colitis 11, 649-670 (2017).

4. $\mathrm{Ng}, \mathrm{S}$. C. et al. Worldwide incidence and prevalence of inflammatory bowel disease in the 21 st century: a systematic review of population-based studies. Lancet 390, 2769-2778 (2017).

5. Halfvarson, J. et al. Dynamics of the human gut microbiome in inflammatory bowel disease. Nat. Microbiol. 2, 1-7 (2017).

6. Dutta, A. K. \& Chacko, A. Influence of environmental factors on the onset and course of inflammatory bowel disease. World J. Gastroenterol. 22, 1088-1100 (2016).

7. Piovani, D. et al. Environmental risk factors for inflammatory bowel diseases: an umbrella review of meta-analyses. Gastroenterology (2019).

8. Davey Smith, G. \& Ebrahim, S. Mendelian randomization ': can genetic epidemiology contribute to understanding environmental determinants of disease ?. Int. J. Epidemiol. https://doi.org/10.1093/ije/dyg070 (2003).

9. Davey Smith, G. \& Hemani, G. Mendelian randomization: genetic anchors for causal inference in epidemiological studies. Hum. Mol. Genet. 23, 1-10 (2014).

10. Burgess, S., Butterworth, A. \& Thompson, S. G. Mendelian randomization analysis with multiple genetic variants using summarized data. Genet. Epidemiol. 37, 658-665 (2013).

11. Collins, R. What makes UK Biobank special?. Lancet 379, 1173-1174 (2012).

12. Liu, M. et al. Association studies of up to 1.2 million individuals yield new insights into the genetic etiology of tobacco and alcohol use. Nat. Genet. 51, 237-244 (2019).

13. Yengo, L. et al. Meta-analysis of genome-wide association studies for height and body mass index in $\sim 700000$ individuals of European ancestry. Hum. Mol. Genet. 27, 3641-3649 (2018).

14. Pulit, S. L. et al. Meta-Analysis of genome-wide association studies for body fat distribution in 694649 individuals of European ancestry. Hum. Mol. Genet. 28, 166-174 (2019).

15. Johansson, M. et al. The influence of obesity-related factors in the etiology of renal cell carcinoma-A mendelian randomization study. PLoS Med. 16, 1-16 (2019).

16. Klimentidis, Y. C. et al. Genome-wide association study of habitual physical activity in over 377,000 UK Biobank participants identifies multiple variants including CADM2 and APOE. Int. J. Obes. 42, 1161-1176 (2018).

17. Thorburn, A. N., Macia, L. \& Mackay, C. R. Diet, metabolites, and 'western-lifestyle' inflammatory diseases. Immunity 40, 833-842 (2014).

18. Zhou, Y. Q., Xu, R. Y. \& Wan, Y. P. The role of dietary factors in inflammatory bowel diseases: new perspectives. J. Dig. Dis. 20, 11-17 (2019).

19. Manousaki, D. et al. Genome-wide association study for Vitamin D levels reveals 69 independent loci. Am. J. Hum. Genet. 106, 327-337 (2020).

20. Grarup, N. et al. Genetic architecture of vitamin B 12 and folate levels uncovered applying deeply sequenced large datasets. PLoS Genet. 9, e10035030 (2013).

21. Kettunen, J. et al. Genome-wide study for circulating metabolites identifies 62 loci and reveals novel systemic effects of LPA. Nat. Commun. 7, 1-9 (2016).

22. de Lange, K. M. et al. Genome-wide association study implicates immune activation of multiple integrin genes in inflammatory bowel disease. Nat. Genet. 49, 256-261 (2017).

23. Burgess, S. Sample size and power calculations in Mendelian randomization with a single instrumental variable and a binary outcome. Int. J. Epidemiol. 43, 922-929 (2014).

24. Burgess, S., Scott, R. A., Timpson, N. J., Davey-smith, G. \& Thompson, S. G. Using published data in Mendelian randomization : a blueprint for efficient identification of causal risk factors. Eur. J. Epidemiol. https://doi.org/10.1007/s10654-015-0011-z (2014).

25. Verbanck, M., Chen, C. Y., Neale, B. \& Do, R. Detection of widespread horizontal pleiotropy in causal relationships inferred from Mendelian randomization between complex traits and diseases. Nat. Genet. 50, 693-698 (2018).

26. Bowden, J., Davey Smith, G. \& Burgess, S. Mendelian randomization with invalid instruments: effect estimation and bias detection through Egger regression. Int. J. Epidemiol. https://doi.org/10.1093/ije/dyv080 (2015).

27. Bowden, J., Smith, G. D., Haycock, P. C. \& Burgess, S. Consistent estimation in Mendelian randomization with some invalid instruments using a weighted median estimator. Genet. Epidemiol. 40, 304-314 (2016)

28. Hartwig, F. P., Smith, G. D. \& Bowden, J. Robust inference in summary data Mendelian randomization via the zero modal pleiotropy assumption. Int. J. Epidemiol. 46, 1985-1998 (2017).

29. Carlens, C. et al. Smoking, use of moist snuff, and risk of chronic inflammatory diseases. Am. J. Respir. Crit. Care Med. 181, 1217-1222 (2010).

30. Ng, S. C. et al. Environmental risk factors in inflammatory bowel disease: a population-based case-control study in Asia-Pacific. Gut 64, 1063-1071 (2015).

31. Singh, S., Dulai, P. S., Zarrinpar, A., Ramamoorthy, S. \& Sandborn, W. J. Obesity in IBD: epidemiology, pathogenesis, disease course and treatment outcomes. Nat. Rev. Gastroenterol. Hepatol. 14, 110-121 (2017).

32. Maruvada, P., Leone, V., Kaplan, L. M. \& Chang, E. B. The human microbiome and obesity: moving beyond associations. Cell Host Microbe 22, 589-599 (2017).

33. Harper, J. W. \& Zisman, T. L. Interaction of obesity and inflammatory bowel disease. World J. Gastroenterol. 22, 7868-7881 (2016).

34. Carreras-Torres, R. et al. Role of obesity in smoking behaviour: Mendelian randomisation study in UK Biobank. BMJ 361, k1767 (2018).

35. Rizzello, F. et al. Implications of the westernized diet in the onset and progression of IBD. Nutrients 11, 1033 (2019).

36. Pan, Y. et al. Associations between folate and vitamin B12 levels and inflammatory bowel disease: a meta-analysis. Nutrients $\mathbf{9}$, $1-15$ (2017).

37. Lund-Nielsen, J. et al. Vitamin D and inflammatory bowel disease: Mendelian randomization analyses in the copenhagen studies and UK Biobank. J. Clin. Endocrinol. Metab. 103, 3267-3277 (2018). 
38. Dixon, L. J., Kabi, A., Nickerson, K. P. \& McDonald, C. Combinatorial effects of diet and genetics on inflammatory bowel disease pathogenesis. Inflamm. Bowel Dis. 21, 912-922 (2015).

39. Ananthakrishnan, A. N. et al. Long-term intake of dietary fat and risk of ulcerative colitis and Crohn's disease. Gut 63, 776-784 (2014).

40. Hart, A. et al. Linoleic acid, a dietary n-6 polyunsaturated fatty acid, and the aetiology of ulcerative colitis: a nested case-control study within a European prospective cohort study. Gut 58, 1606-1611 (2009).

41. Ungaro, F., Rubbino, F., Danese, S. \& D'Alessio, S. Actors and factors in the resolution of intestinal inflammation: lipid mediators as a new approach to therapy in inflammatory bowel diseases. Front. Immunol. 8, 1-13 (2017).

42. Sanna, S. et al. Causal relationships among the gut microbiome, short-chain fatty acids and metabolic diseases. Nat. Genet. 51, 600-605 (2019).

\section{Acknowledgements}

We thank CERCA Program/Generalitat de Catalunya for their institutional support. This project has received funding from the European Union's Horizon 2020 research and innovation programme under the Marie Sklodowska-Curie grant agreement No 796216. MOS received a post-doctoral fellowship from the Spanish Association Against Cancer (AECC) Scientific Foundation. All authors are part of the group 55 of CIBERESP and AGAUR 2017SGR723. We thank all of the participants who took part in the research projects which data were used in this study and the funders and support staff who made this study possible.

\section{Author contributions}

R.C.T., E.J.D. and V.M. conceived of and designed the study. R.C.T. acquired and analysed the data. All authors interpreted the data. R.C.T., G.I.S. and M.O.S. drafted the first version of the article. All authors revised the article critically for important intellectual content and approved the version of the article to be submitted.

\section{Competing interests}

The authors declare no competing interests.

\section{Additional information}

Supplementary information is available for this paper at https://doi.org/10.1038/s41598-020-76361-2.

Correspondence and requests for materials should be addressed to R.C.-T.

Reprints and permissions information is available at www.nature.com/reprints.

Publisher's note Springer Nature remains neutral with regard to jurisdictional claims in published maps and institutional affiliations.

(c) (i) Open Access This article is licensed under a Creative Commons Attribution 4.0 International License, which permits use, sharing, adaptation, distribution and reproduction in any medium or format, as long as you give appropriate credit to the original author(s) and the source, provide a link to the Creative Commons licence, and indicate if changes were made. The images or other third party material in this article are included in the article's Creative Commons licence, unless indicated otherwise in a credit line to the material. If material is not included in the article's Creative Commons licence and your intended use is not permitted by statutory regulation or exceeds the permitted use, you will need to obtain permission directly from the copyright holder. To view a copy of this licence, visit http://creativecommons.org/licenses/by/4.0/.

(C) The Author(s) 2020 